\title{
The effect of breast MRI on disease-free and overall survival in breast cancer patients: a retrospective population-based study
}

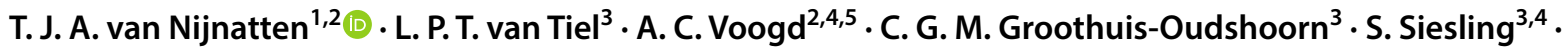 \\ M. B. I. Lobbes ${ }^{1,2,6}$
}

Received: 4 May 2020 / Accepted: 31 August 2020 / Published online: 15 September 2020

(c) The Author(s) 2020

\begin{abstract}
Purpose To evaluate the effect of breast MRI on overall survival (OS) and disease-free survival (DFS) of patients with invasive breast cancer in the Netherlands.

Methods We selected all women from the Netherlands Cancer Registry diagnosed with invasive breast cancer (a) between 2011 and 2013 for the OS-cohort and (b) in the first quarter of 2012 for the DFS-cohort. The study population was subdivided into an MRI and non-MRI group. In addition, subgroups were created according to breast cancer subtype: invasive carcinoma of no special type (NST) versus invasive lobular carcinoma (ILC). OS and DFS were compared between the MRI and non-MRI group using the Kaplan-Meier method and the log-rank test. Cox proportional hazard regression analysis was performed to estimate hazard ratios (HR) with a 95\% confidence interval (CI). To account for missing data, multiple imputation was performed.

Results Of the 31,756 patients included in the OS-cohort (70\% non-MRI and 30\% MRI), 27,752 (87\%) were diagnosed with invasive carcinoma NST and 4004 (13\%) with ILC. Of the 2464 patients included in the DFS-cohort (72\% non-MRI and $28 \%$ MRI), 2161 (88\%) were diagnosed with invasive carcinoma NST and 303 (12\%) with ILC. The distribution of breast MRI use was significantly lower over different age categories, from $49.0 \%$ aged $<50$ to $16.5 \%$ aged $>70$. Multivariable Cox regression showed that breast MRI was not significantly associated with OS overall (HR 0.91, 95\%-CI 0.74-1.11, $p=0.35$ ), nor in the different histological subtypes. Multivariable Cox regression analysis showed that breast MRI was also not significantly associated with DFS (HR 1.16, 95\%-CI 0.81-1.67), nor in the different histological subtypes.

Conclusion Use of breast MRI was not significantly associated with an improved OS or DFS in patients treated with primary surgery.
\end{abstract}

Keywords Breast cancer $\cdot$ Magnetic resonance imaging $\cdot$ Disease-free survival $\cdot$ Overall survival

\section{Introduction}

Breast cancer is the second most common cancer-type in the world (2.09 million cases) and for women it is the most common cancer-type [1]. Annually, more than 15,000 Dutch

T. J. A. van Nijnatten, L. P. T. van Tiel, S. Siesling and M. B. I. Lobbes have contributed equally to this work.

Electronic supplementary material The online version of this article (https://doi.org/10.1007/s10549-020-05906-w) contains supplementary material, which is available to authorized users.

T. J. A. van Nijnatten

Thiemovn@gmail.com

Extended author information available on the last page of the article women are diagnosed with breast cancer, the most frequent type of cancer among women in the Netherlands [2].

Conventional imaging techniques for early detection and diagnosis of breast cancer are full-field digital mammography (FFDM) and ultrasound, in combination with tissue sampling of suspicious lesions [3]. In the last decades, an increase is observed in the use of (preoperative) breast magnetic resonance imaging (MRI) as an additional diagnostic imaging technique, in particularly applied in cases where the therapeutic pathway is still questioned based upon conventional imaging. However, the use of breast MRI has become a subject of debate as several studies questioned its additional value, even arguing that it might result in more mastectomies [4]. Theoretically, breast MRI could have beneficial effects, because of its superior ability to assess for 
instance maximum tumour diameter, multifocality, and presence of contralateral breast cancer [5, 6]. Therefore, breast MRI could be used for optimizing the extent of surgery and radiotherapy, which may reduce the risk of involved surgical margins and the need for re-excision, moreover it may result in improved local control [7], which in turn might lead to fewer metastases, and an improved disease-free (DFS) and overall survival (OS). On the other hand, due to the high sensitivity of MRI, the possibility of overdiagnosis exists and it might even result in a higher risk of false-positive results [3]. Both may lead to unnecessary and/or more extensive resections, treatment delay, and higher costs $[8,9]$.

Regarding short-term effects of the use of breast MRI, previous studies have shown that mastectomy rates increased, and that the risk of positive surgical margins and re-excision rates showed no or only a slight improvement $[3,4,8]$. However, for some subgroups, such as invasive lobular carcinoma (ILC), breast MRI might have beneficial short-term effects, but published results are contradictory $[3,4,8]$. Regarding the long-term effects of breast MRI, previous studies have shown that there were no statistically significant differences in the risk of local and distant recurrences, contralateral breast cancer development, and diseasefree and overall survival [7, 9-14]. However, some studies indicated that breast MRI has a tendency towards improved survival $[11,14]$. Hence, there are still uncertainties about whether breast MRI has a beneficial effect on the long-term outcome. The purpose of this retrospective population-based study was to evaluate the effect of the use of breast MRI on the overall and disease-free survival of patients with invasive breast cancer in the Netherlands.

\section{Methods}

\section{Data collection}

This retrospective study included all female patients treated with surgery for invasive breast carcinoma of no special type (NST) or ILC, diagnosed in the period of 2011-2013 in the Netherlands. The OS had a maximum follow-up of 7 years. Data on recurrent disease for the DFS-cohort were available for patients diagnosed and treated in the first three months of 2012, and had a maximum follow-up of 5 years. No agelimits were applied and patients treated with adjuvant radiotherapy, chemotherapy, hormonal and/or target therapies were included. Patients who did not undergo surgical treatment, who had distant metastases at baseline, and/or who had received neo-adjuvant therapy were excluded.

Data were obtained from the Netherlands Cancer Registry (NCR), and were registered by trained registrars based on notification by the automated pathology laboratory archive (PALGA). The 7th edition of the TNM-classification was used [15]. According to national guidelines, routine use of breast MRI was not recommended, but could be considered in these indications: (a) patients with ILC, (b) patients with invasive carcinoma who preferred breast conserving surgery but who had discrepancies in tumour size assessment between physical examination and imaging. These recommendations applied in particular to young women [16].

The following variables were selected for the present study: age at diagnosis (categorized in $<50,50-59,60-69$, or $\geq 70$ years), tumour status (T1, T2, or T3-4), nodal status (N0, N1, N2, or N3), molecular subtypes (ER/PR + HER2-: estrogen receptor (ER) and/or progesterone receptor (PR) positive, HER2 negative; ER/PR + HER2 + : ER and/or PR positive, HER2 positive; HER2 enriched: ER and PR negative, HER2 positive; and triple negative: ER, PR, and HER2 negative), histological grade (low, medium, or high), multifocality (yes or no), tumour location (lateral, medial, or other), breast MRI use (yes or no), surgical margin status after surgery (negative margin $(\mathrm{NM})<0 \mathrm{~mm}$, focal positive margin $(\mathrm{FPM})<4 \mathrm{~mm}$, or more than focal positive margin $($ MFPM) $>4 \mathrm{~mm}$ ), type of final surgery (mastectomy or breast conserving surgery), adjuvant therapy (radiotherapy, chemotherapy, hormonal or targeted treatment), and vital status (date of death). For patients diagnosed and treated in the first quarter of 2012, active follow-up was conducted whereby information on the date of local recurrence (yes or no), regional recurrence (yes or no), and distant metastases (yes or no) was collected. Time period until death or last contact was linked with the database of the municipality, and was updated until February 1st, 2018.

\section{Statistical analysis}

The study population was divided into an MRI and non-MRI group, according to the use of breast MRI. Subsequently, the study population was stratified into one of the following histological subgroups: invasive carcinoma NST or ILC. General characteristics between the groups were tested using the Chi-square test for categorical variables and the Mann-Whitney $U$ test for continuous variables. OS and DFS were calculated with the Kaplan-Meier method and its survivor function, which predicts the chance of survival after a given period of time. Kaplan-Meier survival curves were compared with the log-rank test. For the DFS an event was defined as any local recurrence (LR), regional recurrence (RR), or distant metastasis (DM) within 5 years after the primary diagnosis. Possible confounders were examined using univariable and multivariable Cox proportional hazard regression analysis, with hazard ratio (HR) and corresponding $95 \%$ confidence interval (CI). In the multivariable model of the OS analysis, the interaction term MRI and age per category was included to test whether the effect of MRI differs between age category. Multiple imputation by the chained 
equations, with 50 iterations and 20 imputations, was used to account for missing data [17]. The pooled results were used based on Rubin's rule. Variables in the multivariable model, that were not statistically significant, were excluded based on significance and the Akaike's information criterion (AIC) selection method. Proportional hazards assumption was tested by the Schoenfeld test and by plotting the scaled Schoenfeld residuals. Statistical analyses were performed by using Stata/SE version 14.2 (StataCorp. LP, College Station, TX, USA). $P$-Values (two-sided) less than 0.05 were considered statistically significant.

\section{Results}

\section{General characteristics}

In the period 2011-2013 31,877 patients were treated with surgery for invasive carcinoma NST or ILC. Patients with no follow-up data $(n=80)$, unknown surgery $(n=20)$, or an unknown adjuvant treatment $(n=21)$ were excluded. After exclusion of these patients 31,756 (99.6\%) patients composed the OS-cohort for the analysis of the OS. Of these patients 22,124 (70\%) did not undergo breast MRI, $27,752(87 \%)$ had invasive carcinoma NST (73\% non-MRI and 27\% MRI) and 4004 (13\%) had ILC (44\% non-MRI and $56 \%$ MRI). The final study cohort for the DFS (DFScohort) included 2464 patients, of whom 1767 (72\%) did not undergo breast MRI. Of these patients, $2161(88 \%)$ had invasive carcinoma NST (75\% non-MRI and 25\% MRI) and 303 (12\%) had ILC (46\% non-MRI and 54\% MRI).

Table 1 shows patient, tumour, and treatment characteristics of the OS-cohort, according to breast MRI use. Patients in the non-MRI group were older compared to the MRI group (62.9 vs 56.1 years, respectively) and were less often treated with adjuvant chemotherapy, hormonal and/or target therapies. In addition, the percentage of patients who underwent breast MRI was lower in higher age categories (from $49.0 \%$ in age $<50$ until $16.5 \%$ in age $>70$ ). The MRI group had slightly larger tumours, were more likely to have axillary lymph node involvement and multifocal tumours, and more often underwent mastectomy as the final surgical procedure. The histological subgroups had similar patient characteristics, except for the ILC subgroup, where, in general, more mastectomies as final surgery were performed. Regarding the involvement of surgical margins after breast conserving surgery, there was no statistically significant difference between the MRI and non-MRI group for the total study population $(p=0.08)$ and the invasive carcinoma NST $(p=0.39)$. For the ILC subgroup a statistically significant difference was observed $(p=0.002)$; here the MRI group was more likely to have negative margins (79\% versus $73 \%)$, and less likely to have more than focal positive margins ( $8 \%$ versus $12 \%$ ), compared to the non-MRI group.

There were 2939 (9\%) incomplete cases in the OS-cohort (most frequent missing case: histological grade $(3.3 \%, 1060$ observations)) and 231 (9.0\%) in the DFS-cohort (most frequent missing case: histological grade $(3.6 \%, 89$ observations)) for which multiple imputation was used, assuming that the data were missing at random. The imputed data were compared with the original data, which showed an overall comparable distribution of the data (Online Appendix 1, Tables 1 and 2).

\section{Overall survival}

In the total OS-cohort $(n=31,756), 2938(13.0 \%)$ of the 22,124 patients in the non-MRI group died, compared to $743(8.0 \%)$ of the 9632 patients in the MRI group, after a mean follow-up of 5.3 years (range $0.1-7.1$ years, see Table 3 in Online Appendix 2 for the events of deaths per subgroup, stratified by age categories). The Kaplan-Meier analysis and the log-rank test showed that patients in the MRI group had a significantly better OS than the patients in the non-MRI group $(p<0.0001)$; the 7-year survival rates were 0.89 (95\%-CI 0.87-0.90) and 0.83 (95\%-CI 0.82-0.83), respectively (Online Appendix 3). However, after age stratification in the total study population, this difference in OS proved to be only statistically significant for the age groups 60-69 $(p=0.036)$ and $>70(p<0.0001)$. In Figs. 1 and 2 the Kaplan-Meier curves are shown per histological subgroup and stratified by age category.

The multivariable analysis of the OS regarding breast MRI use demonstrated no statistical differences among different age categories for the effect of breast MRI, neither in the overall OS-cohort, nor in the histological subgroups (total study population: $p=0.06$; invasive carcinoma NST: $p=0.07$; ILC: $p=0.23$ ) (Table 3).

\section{Disease-free survival}

Within the DFS-cohort ( $n=2464), 114(7.0 \%)$ of 1767 patients in the non-MRI group had an event compared to $48(7.0 \%)$ of 697 in the MRI group, after a mean followup of 4.6 years. Table 2 provides an overview of the number of disease-related events after five years, according to MRI use. The Kaplan-Meier analysis and the log-rank test showed that undergoing a breast MRI was not significantly associated with DFS $(p=0.84)$. The 5-year DFS rates was 0.93 (95\%-CI 0.91-0.95) for the MRI group vs 0.93 (95\%CI 0.92-0.94) in the non-MRI group (Online Appendix 3, Table 5). In both the invasive carcinoma NST and ILC subgroups, breast MRI use was also not significantly associated with DFS. In the invasive carcinoma NST group the 5-year survival rate was $0.93(95 \%$-CI $0.90-0.94)$ for the 
Table 1 Patient, tumour, and treatment characteristics total study population and per subgroup, according to use of breast MRI

\begin{tabular}{|c|c|c|c|c|c|c|c|c|c|c|c|c|}
\hline \multirow[t]{3}{*}{ Characteristic } & \multicolumn{4}{|c|}{ Total study population } & \multicolumn{4}{|c|}{ Invasive carcinoma NST } & \multicolumn{4}{|l|}{ ILC } \\
\hline & \multicolumn{2}{|c|}{ non-MRI $(n=22,124)$} & \multicolumn{2}{|c|}{ MRI $(n=9632)$} & \multicolumn{2}{|c|}{ non-MRI $(n=20,366)$} & \multicolumn{2}{|c|}{ MRI $(n=7386)$} & \multicolumn{2}{|c|}{ non-MRI $(n=1758)$} & \multicolumn{2}{|c|}{$\operatorname{MRI}(n=2246)$} \\
\hline & $n$ & $(\%)$ & $n$ & $(\%)$ & $n$ & $(\%)$ & $n$ & $(\%)$ & $n$ & $(\%)$ & $n$ & $(\%)$ \\
\hline \multicolumn{13}{|l|}{ Age } \\
\hline Mean (range) & 62.9 & $(19-97)$ & 56.1 & $(21-94)$ & 62.5 & $(19-97)$ & 54.8 & $(21-94)$ & 66.9 & $(28-93)$ & 60.3 & $(24-88)$ \\
\hline$<50$ & 3092 & (14) & 2976 & $(30.9)$ & 2936 & $(14.4)$ & 2557 & $(34.6)$ & 156 & $(8.9)$ & 419 & $(18.7)$ \\
\hline $50-59$ & 5375 & $(24.3)$ & 2821 & $(29.3)$ & 5083 & $(25)$ & 2168 & $(29.4)$ & 292 & $(16.6)$ & 653 & $(29.1)$ \\
\hline $60-69$ & 7078 & (32) & 2534 & $(26.3)$ & 6529 & $(32.1)$ & 1834 & $(24.8)$ & 549 & $(31.2)$ & 700 & $(31.2)$ \\
\hline$>70$ & 6579 & (29.7) & 1301 & (13.5) & 5818 & $(28.6)$ & 827 & (11.2) & 761 & (43.3) & 474 & $(21.1)$ \\
\hline \multicolumn{13}{|l|}{ Tumour status } \\
\hline 1 & 15,366 & $(69.8)$ & 6223 & $(65)$ & 14,444 & $(71.2)$ & 5072 & $(69.1)$ & 922 & $(52.7)$ & 1151 & $(51.5)$ \\
\hline 2 & 6115 & $(27.8)$ & 2919 & $(30.5)$ & 5446 & $(26.9)$ & 2074 & $(28.2)$ & 669 & $(38.2)$ & 845 & $(37.8)$ \\
\hline $3-4$ & 547 & $(2.5)$ & 436 & $(4.6)$ & 388 & (1.9) & 199 & $(2.7)$ & 159 & $(9.1)$ & 237 & $(10.6)$ \\
\hline Unknown & 96 & & 54 & & 88 & & 41 & & 8 & & 13 & \\
\hline \multicolumn{13}{|l|}{ Nodal status } \\
\hline 0 & 14,831 & $(68.5)$ & 6168 & $(64.7)$ & 13,748 & $(69)$ & 4736 & $(64.8)$ & 1083 & $(63.5)$ & 1432 & $(64.5)$ \\
\hline 1 & 5313 & $(24.6)$ & 2547 & (26.7) & 4886 & $(24.5)$ & 1989 & $(27.2)$ & 427 & (25) & 558 & $(25.1)$ \\
\hline 2 & 928 & $(4.3)$ & 527 & $(5.5)$ & 842 & $(4.2)$ & 396 & $(5.4)$ & 86 & (5) & 131 & $(5.9)$ \\
\hline 3 & 566 & $(2.6)$ & 288 & (3) & 456 & $(2.3)$ & 188 & $(2.6)$ & 110 & $(6.4)$ & 100 & $(4.5)$ \\
\hline Unknown & 486 & & 102 & & 434 & & 77 & & 52 & & 25 & \\
\hline \multicolumn{13}{|l|}{ Histological grade } \\
\hline Low & 5417 & $(25.3)$ & 2112 & $(22.8)$ & 5167 & $(26.2)$ & 1726 & $(24.2)$ & 250 & $(14.9)$ & 386 & (18) \\
\hline Medium & 9690 & $(45.2)$ & 4614 & $(49.8)$ & 8423 & $(42.7)$ & 3023 & $(42.4)$ & 1267 & $(75.3)$ & 1591 & (74.1) \\
\hline High & 6316 & $(29.5)$ & 2547 & $(27.5)$ & 6150 & $(31.2)$ & 2378 & $(33.4)$ & 166 & $(9.9)$ & 169 & $(7.9)$ \\
\hline Unknown & 701 & & 359 & & 626 & & 259 & & 75 & & 100 & \\
\hline \multicolumn{13}{|l|}{ Multifocal } \\
\hline No & 19,579 & $(89.5)$ & 7305 & $(76.1)$ & 18,118 & $(89.9)$ & 5630 & $(76.5)$ & 1461 & $(84.8)$ & 1675 & (74.9) \\
\hline Yes & 2300 & $(10.5)$ & 2289 & $(23.9)$ & 2039 & $(10.1)$ & 1728 & $(23.5)$ & 261 & $(15.2)$ & 561 & $(25.1)$ \\
\hline Unknown & 245 & & 38 & & 209 & & 28 & & 36 & & 10 & \\
\hline \multicolumn{13}{|l|}{ Molecular subtype } \\
\hline ER/PR + HER2- & 16,531 & $(77.3)$ & 7376 & $(78.3)$ & 14,976 & (76) & 5300 & $(73.3)$ & 1555 & (93) & 2076 & $(94.4)$ \\
\hline $\mathrm{ER} / \mathrm{PR}+\mathrm{HER} 2+$ & 1707 & (8) & 836 & $(8.9)$ & 1651 & $(8.4)$ & 757 & $(10.5)$ & 56 & $(3.3)$ & 79 & $(3.6)$ \\
\hline HER2 enriched & 808 & $(3.8)$ & 372 & $(3.9)$ & 801 & $(4.1)$ & 359 & (5) & 7 & $(0.4)$ & 13 & $(0.6)$ \\
\hline Triple negative & 2327 & $(10.9)$ & 842 & $(8.9)$ & 2273 & (11.5) & 811 & $(11.2)$ & 54 & $(3.2)$ & 31 & $(1.4)$ \\
\hline Unknown & 751 & & 206 & & 665 & & 159 & & 86 & & 47 & \\
\hline \multicolumn{13}{|l|}{ Tumour location } \\
\hline Lateral & 10,708 & $(49.0)$ & 4302 & $(45.2)$ & 9863 & $(49.0)$ & 3340 & $(45.7)$ & 845 & $(48.9)$ & 962 & $(43.4)$ \\
\hline Medial & 4693 & $(21.5)$ & 1830 & $(19.2)$ & 4410 & $(21.9)$ & 1455 & (19.9) & 283 & $(16.4)$ & 375 & $(16.9)$ \\
\hline Other & 6462 & $(29.6)$ & 3389 & $(35.6)$ & 5861 & $(29.1)$ & 2510 & $(34.4)$ & 601 & $(34.8)$ & 879 & $(39.7)$ \\
\hline Unknown & 261 & & 111 & & 232 & & 81 & & 29 & & 30 & \\
\hline \multicolumn{13}{|l|}{ Final operation } \\
\hline Mastectomy & 7987 & $(36.1)$ & 4717 & $(49)$ & 6981 & $(34.3)$ & 3441 & $(46.6)$ & 1006 & $(57.2)$ & 1276 & $(56.8)$ \\
\hline BCS & 14,137 & $(63.9)$ & 4915 & $(51)$ & 13,385 & $(65.7)$ & 3945 & $(53.4)$ & 752 & $(42.8)$ & 970 & $(43.2)$ \\
\hline \multicolumn{13}{|l|}{ Surgical margin $¥$} \\
\hline NM & 20,207 & $(93.2)$ & 8955 & $(93.7)$ & 18,650 & $(93.4)$ & 6899 & $(94.1)$ & 1557 & (91.7) & 2056 & $(92.4)$ \\
\hline FPM & 1286 & $(5.9)$ & 530 & $(5.5)$ & 1174 & $(5.9)$ & 391 & $(5.3)$ & 112 & $(6.6)$ & 139 & $(6.3)$ \\
\hline MFPM & 178 & $(0.8)$ & 73 & $(0.8)$ & 149 & $(0.7)$ & 44 & $(0.6)$ & 29 & $(1.7)$ & 29 & $(1.3)$ \\
\hline Unknown & 453 & & 74 & & 393 & & 52 & & 60 & & 22 & \\
\hline
\end{tabular}


Table 1 (continued)

\begin{tabular}{|c|c|c|c|c|c|c|c|c|c|c|c|c|}
\hline \multirow[t]{3}{*}{ Characteristic } & \multicolumn{4}{|c|}{ Total study population } & \multicolumn{4}{|c|}{ Invasive carcinoma NST } & \multicolumn{4}{|l|}{ ILC } \\
\hline & \multicolumn{2}{|c|}{ non-MRI $(n=22,124)$} & \multicolumn{2}{|c|}{ MRI $(n=9632)$} & \multicolumn{2}{|c|}{ non-MRI $(n=20,366)$} & \multicolumn{2}{|c|}{ MRI $(n=7386)$} & \multicolumn{2}{|c|}{ non-MRI $(n=1758)$} & \multicolumn{2}{|c|}{$\operatorname{MRI}(n=2246)$} \\
\hline & $n$ & $(\%)$ & $n$ & $(\%)$ & $n$ & (\%) & $n$ & $(\%)$ & $n$ & $(\%)$ & $n$ & (\%) \\
\hline \multicolumn{13}{|l|}{$\overline{\text { Adjuvant therapy }}$} \\
\hline Radio-yes & 15,456 & $(69.9)$ & 6129 & (63.6) & 14,452 & (71) & 4728 & (64) & 1004 & (57.1) & 1401 & (62.4) \\
\hline Chemo-yes & 7466 & (33.7) & 4760 & $(49.4)$ & 7031 & $(34.5)$ & 3,796 & (51.4) & 435 & $(24.7)$ & 964 & $(42.9)$ \\
\hline Hormonal-yes & 11,946 & (54) & 5995 & $(62.2)$ & 10,678 & $(52.4)$ & 4267 & $(57.8)$ & 1268 & $(72.1)$ & 1728 & (76.9) \\
\hline Target-yes & 1643 & (7.4) & 969 & $(10.1)$ & 1605 & $(7.9)$ & 902 & $(12.2)$ & 38 & $(2.2)$ & 67 & (3) \\
\hline
\end{tabular}

$N S T$ no special type, $I L C$ invasive lobular carcinoma, $M R I$ magnetic resonance imaging, $n$ number of patients, $¥$ final operation, $B C S$ breast conserving surgery, $N M$ negative margin, $F P M$ focal positive margin, $M F P M$ more than focal positive margin

Table 2 Overview event and event type per (sub)group in DFS-cohort after 5 years

\begin{tabular}{|c|c|c|c|c|c|c|}
\hline & \multicolumn{2}{|c|}{ Total study population } & \multicolumn{2}{|c|}{ Invasive carcinoma NST } & \multicolumn{2}{|l|}{ ILC } \\
\hline & non-MRI (\%) & MRI (\%) & non-MRI (\%) & MRI (\%) & non-MRI (\%) & MRI (\%) \\
\hline \multicolumn{7}{|l|}{ Event } \\
\hline Yes & $114(6.5)$ & $48(6.9)$ & $103(6.3)$ & $39(7.3)$ & $11(7.9)$ & $9(5.5)$ \\
\hline No & $1653(93.5)$ & $649(93.1)$ & 1524 (93.7) & 495 (92.7) & $129(92.1)$ & $154(94.5)$ \\
\hline \multicolumn{7}{|l|}{ Event type } \\
\hline LR & $6(0.4)$ & $8(1.2)$ & $6(0.4)$ & $7(1.3)$ & 0 & $1(0.6)$ \\
\hline $\mathrm{RR}$ & $10(0.6)$ & $2(0.3)$ & $10(0.6)$ & $2(0.4)$ & 0 & 0 \\
\hline DM & $67(3.8)$ & $29(4.2)$ & $61(3.7)$ & $22(4.1)$ & $6(4.3)$ & $7(4.3)$ \\
\hline $\mathrm{LR}+\mathrm{RR}$ & $6(0.4)$ & $2(0.3)$ & $5(0.3)$ & $1(0.2)$ & $1(0.7)$ & $1(0.6)$ \\
\hline $\mathrm{LR}+\mathrm{DM}$ & $6(0.4)$ & $1(0.1)$ & $5(0.3)$ & $1(0.2)$ & $1(0.7)$ & 0 \\
\hline $\mathrm{RR}+\mathrm{DM}$ & $14(0.8)$ & $6(0.9)$ & $12(0.7)$ & $6(1.1)$ & $2(1.4)$ & 0 \\
\hline $\mathrm{LR}+\mathrm{RR}+\mathrm{D} \mathrm{M}$ & $5(0.3)$ & 0 & $4(0.2)$ & 0 & $1(0.7)$ & 0 \\
\hline
\end{tabular}

NST no special type, ILC invasive lobular carcinoma, MRI magnetic resonance imaging, $L R$ local recurrence, $R R$ regional recurrence, $D M$ distant metastasis
MRI group and 0.93 (95\%-CI 0.92-0.95) for the non-MRI group $(p=0.54)$. In the ILC subgroup these figures were 0.94 (95\%-CI 0.89-0.97) and 0.92 (95\%-CI 0.86-0.95), respectively $(p=0.36)$.

The multivariable analysis showed no statistically significant difference in DFS regarding breast MRI use, neither in the total DFS-cohort, nor in the histological subgroups (total study population: $p=0.42$; invasive carcinoma NST: $p=0.32$; ILC: $p=0.96$ ) (Table 3).

\section{Discussion}

The purpose of this retrospective population-based study was to evaluate the association of breast MRI use on survival for invasive breast cancer patients treated with primary surgery in the Netherlands. Multivariable Cox regression showed that the effect of breast MRI on OS is not statistical different among different age categories $(<50$ years, 50-59 years, 60-69 years and $>70$ years, respectively), neither in the overall OS-cohort, nor in the histological subgroups. Yet, the use of breast MRI decreased dramatically from $49.0 \%$ in patients aged $<50$ to $16.5 \%$ in patients aged $>70$. Regarding the DFS-cohort, Multivariable Cox regression showed also no statistically significant difference in DFS between patients with or without breast MRI.

In contrast to our study, which demonstrated no significant effect of breast MRI use with regard to overall survival, a tendency towards positive correlation of breast MRI use on OS was observed in previous studies, but this association was not statistically significant [7, 11-14]. Ryu and colleagues indicated in a study of clinically T1-2 breast cancer patients that breast MRI use was not associated with a better OS (HR 1.18 95\%-CI 0.27-5.08) [12]. Solin et al. indicated in a non-randomized retrospective analysis that there were no differences between the two groups for OS (univariable HR 0.84, 95\%-CI 0.50-1.41, $p=0.51$ ), however many patients underwent breast MRI after surgery within this study cohort [13]. In a non-randomized retrospective study towards early stage invasive carcinomas treated with breast conservation treatment (BCT), with a median followup of 13.8 years, Vapiwala et al. showed that breast MRI use 

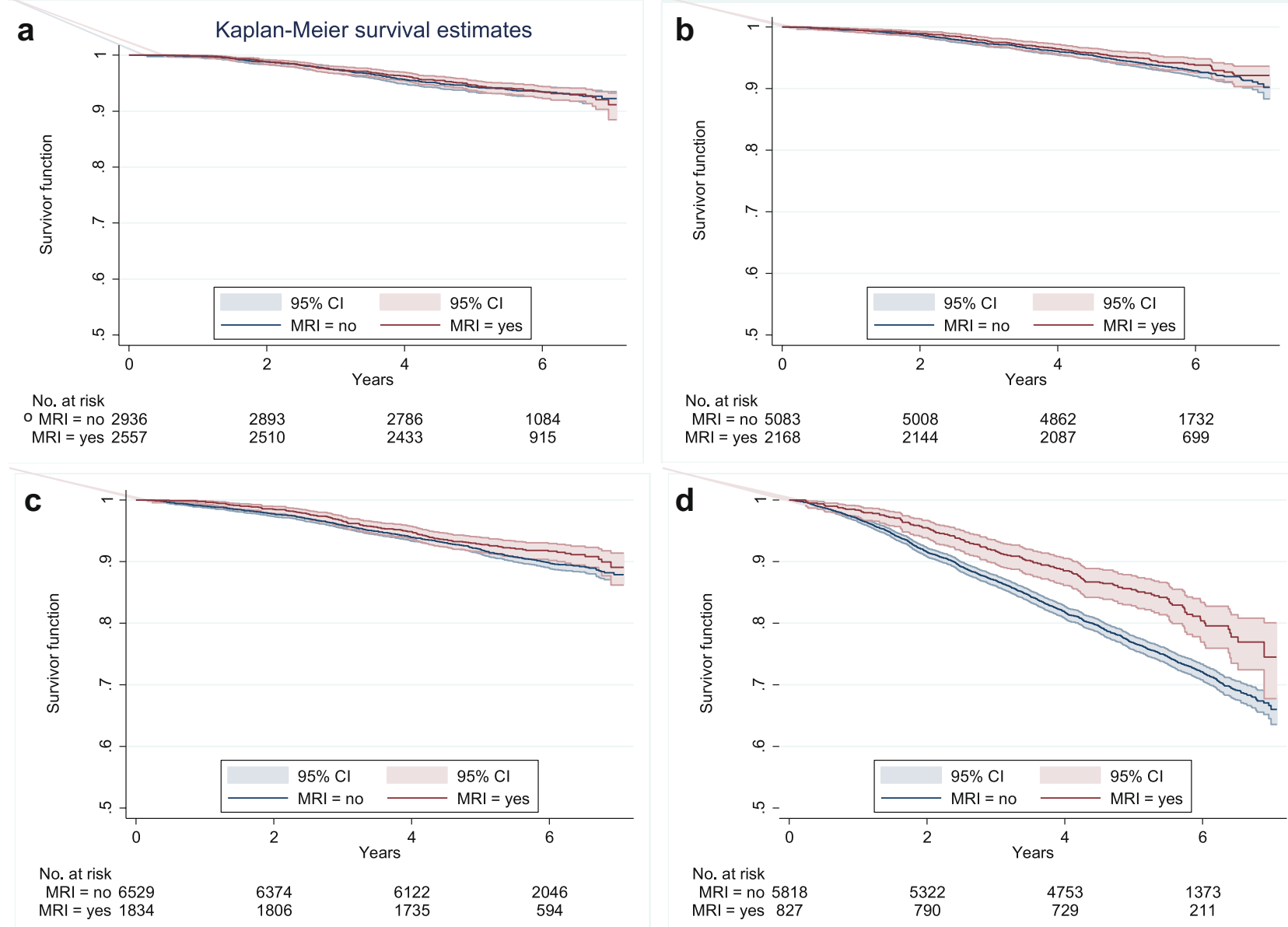

a. age $<50$ years. b. age $50-59$ years. c. age $60-69$ years. $d$. age $>70$ years.

Fig. 1 Kaplan-Meier curve of overall survivor function subgroup invasive carcinoma NST, stratified per age category $(2011-2013, n=27,752$; $\left.n_{\mathrm{a}}=5493, n_{\mathrm{b}}=7251, n_{\mathrm{c}}=8363, n_{\mathrm{d}}=6645\right)$

had no significant impact on 15 year OS (MRI group 77\% vs $71 \%$ non-MRI group, $p=0.24$ ) [7]. Choi et al. found that the MRI group had a tendency towards better survival, however insignificant (univariable HR 0.79, 95\%-CI 0.48-1.31, $p=0.362$ ) [11]. Ha et al. studied the effect of breast MRI solely on ILC patients. Their results showed no statistical significance with the use of breast MRI or not regarding OS (HR 0.485, 95\%-CI 0.149-1.585, $p=0.231$ ) [14], which is in line with our results.

Although previously mentioned studies observed similar results when compared to our study, these studies were limited by heterogeneous data and several impairments. For instance multivariable analyses without including breast MRI [11], unequal follow-up time between both groups (MRI vs non-MRI) [12] or missing results of the multivariable models [13]. In addition, three of the previously mentioned studies focused solely on patients undergoing BCT $[7,12,13]$ and two studies included both patients with breast conserving surgery as mastectomy $[11,14]$. All studies were based on patients cohorts from single institutions, which may limit the generalizability of their findings [7, 11-14]. In addition, the study populations were significant smaller than our study population (range 287-2441) and patient characteristics were not completely balanced, the MRI group were younger and had slightly more favourable tumour characteristics, indicating that our study population seems a more balanced study population when compared to previously performed studies [7, 13, 14].

Surgical margin in patients treated with breast conservative surgery is an important prognostic factor as it is known to affect DFS in women who did not undergo breast MRI [9]. According to our results, the use of breast MRI resulted in statistically significant more frequent cancer-free surgical margins in the case of ILC (79\% versus $73 \%, p=0.002$ ), but not in the subgroup of invasive carcinoma NST $(p=0.39)$. In contrast to our results, Lai et al. showed an overall increased risk of surgical margin involvement when breast MRI was omitted [18]. This 

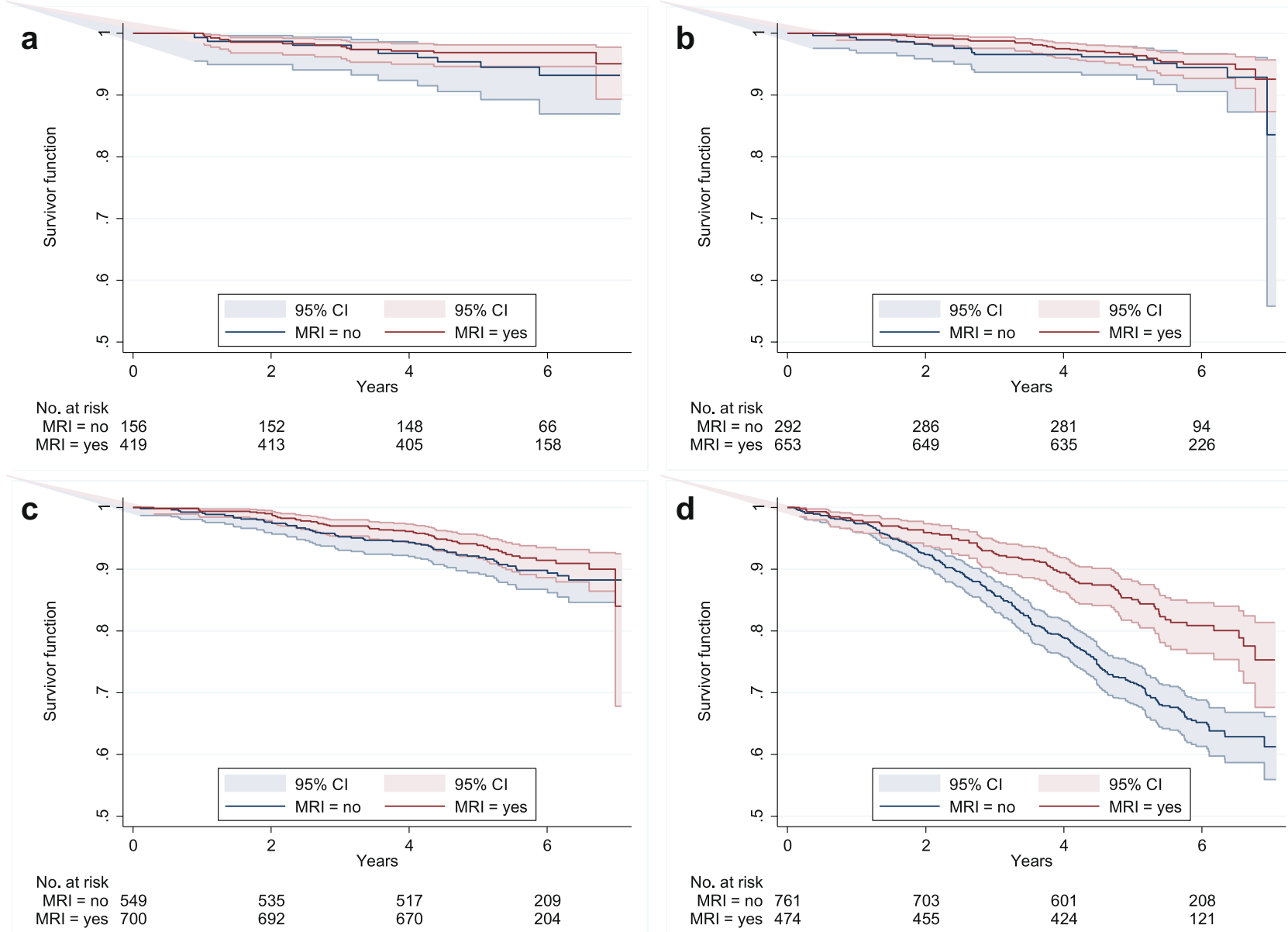

a. age $<50$ years. b. age 50-59 years. c. age 60-69 years. d. age $>70$ years.

Fig. 2 Kaplan-Meier curve of overall survivor function subgroup ILC, stratified per age category $\left(2011-2013, n=4004 ; n_{\mathrm{a}}=575, n_{\mathrm{b}}=945\right.$, $\left.n_{\mathrm{c}}=1249, n_{\mathrm{d}}=1235\right)$

could be explained, among other things, by the inclusion of patients diagnosed with DCIS only in the study of Lai et al. in contrast to our cohort including patients diagnosed with invasive breast cancer only. Consequently, the percentages of radical surgical margins differed among the studies (88.7\% [18] versus $83.9 \%$ in our study).

This study has several strengths and limitations. One strength is the use of a nationwide population-based cancer registry, which increases the generalizability of the results. This also led to a large sample size, which made stratification on the subgroups invasive carcinoma NST and ILC, and the age categories possible. However, since the sample size was large, the confidence intervals were relatively small which might lead to statistically significant results, yet not clinically relevant. It is important to take this in consideration while interpreting the results. Another strength of this study is that it includes several other prospective factors which may influence and adjust the HR of breast MRI, such as the tumour location, type of operation and surgical margins.

A limitation of this study is the retrospective and observational design. This reflects daily practice which leads to a large difference in distribution of breast MRI use among the different age categories in our study. The use of breast MRI decreased with increasing age, especially in patients $60-70$ and $>70$. The use of MRI might be affected by factors which we did not have any information on in the NCR, such as the presence of comorbid conditions in patients. Another important limitation is that the reason to perform an MRI and whether the MRI findings changed the original surgical treatment plan or not were unknown, which leaves room for confounding by indication. Besides, the number of surgical excisions to achieve negative margins per patient in the current study was unknown. Next, for this study patients treated with neo-adjuvant systemic therapy were excluded. Yet, this subgroup of patients is considered to be an important 
Table 3 Results of multivariable Cox proportional hazard regression analysis, for both OS and DFS, respectively for total study population (a), invasive carcinoma NST (b) and ILC (c)

\begin{tabular}{|c|c|c|c|c|c|c|}
\hline \multirow[t]{3}{*}{ Variable } & \multicolumn{3}{|c|}{ Overall survival } & \multicolumn{3}{|c|}{ Disease-free survival } \\
\hline & \multicolumn{3}{|c|}{ Total study population } & \multicolumn{3}{|c|}{ Total study population } \\
\hline & HR & $95 \%$-CI & $P$-value & HR & 95\%-CI & $P$-value \\
\hline \multicolumn{7}{|l|}{$\mathrm{a}$} \\
\hline MRI & & & - & & & - \\
\hline Yes & 0.91 & $(0.74-1.11)$ & 0.35 & 1.16 & $(0.81-1.67)$ & 0.42 \\
\hline Age & & & $<0.01 *$ & & & $0.29 *$ \\
\hline$<50$ & 1 & - & & 1 & - & \\
\hline $50-59$ & 3.52 & $(2.98-4.16)$ & $<0.01$ & 1.35 & $(0.79-2.29)$ & 0.27 \\
\hline $60-69$ & 1.25 & $(1.05-1.49)$ & 0.01 & 1.55 & $(0.91-2.66)$ & 0.11 \\
\hline$>70$ & 1.76 & $(1.49-2.08)$ & $<0.01$ & 1.83 & $(0.97-3.45)$ & 0.06 \\
\hline MRI\#age & & & $0.06^{*}$ & & & \\
\hline$<50$ & 1 & - & & - & - & \\
\hline $50-59$ & 0.88 & $(0.67-1.17)$ & 0.38 & - & - & \\
\hline $60-69$ & 0.88 & $(0.68-1.15)$ & 0.36 & - & - & \\
\hline$>70$ & 0.73 & $(0.57-0.93)$ & 0.01 & - & - & \\
\hline Tumour status & & & $<0.01 *$ & & & $<0.01 *$ \\
\hline 1 & 1 & - & & 1 & - & \\
\hline 2 & 1.69 & $(1.56-1.83)$ & $<0.01$ & 2.56 & $(1.74-3.78)$ & $<0.01$ \\
\hline $3-4$ & 2.30 & $(1.99-2.66)$ & $<0.01$ & 3.86 & $(2.00-7.46)$ & $<0.01$ \\
\hline Nodal status & & & $<0.01^{*}$ & & & $<0.01 *$ \\
\hline 0 & 1 & - & & 1 & - & \\
\hline 1 & 1.66 & $(1.52-1.80)$ & $<0.01$ & 1.70 & $(1.14-2.55)$ & 0.01 \\
\hline 2 & 3.46 & $(3.03-3.95)$ & $<0.01$ & 4.75 & $(2.68-8.41)$ & $<0.01$ \\
\hline 3 & 6.01 & $(5.22-6.92)$ & $<0.01$ & 10.59 & $(6.09-18.43)$ & $<0.01$ \\
\hline Hist. grade & & & $<0.01 *$ & & & $<0.01 *$ \\
\hline Low & 1 & - & & 1 & - & \\
\hline Medium & 1.27 & $(1.14-1.41)$ & $<0.01$ & 1.80 & $(0.96-3.36)$ & 0.07 \\
\hline High & 1.97 & $(1.75-2.21)$ & $<0.01$ & 4.19 & $(2.20-7.97)$ & $<0.01$ \\
\hline \multicolumn{7}{|l|}{ Multifocal } \\
\hline Yes & $£$ & - & & $£$ & - & \\
\hline Mol. subtype & & & $0.01 *$ & & & $0.53^{*}$ \\
\hline ER/PR + HER2- & 1 & - & & 1 & - & \\
\hline $\mathrm{ER} / \mathrm{PR}+\mathrm{HER} 2+$ & 1.23 & $(1.06-1.42)$ & $<0.01$ & 1.51 & $(0.78-2.90)$ & 0.22 \\
\hline HER2 enr & 1.41 & $(1.17-1.69)$ & $<0.01$ & 1.68 & $(0.70-4.00)$ & 0.25 \\
\hline Triple neg & 1.74 & $(1.54-1.96)$ & $<0.01$ & 1.26 & $(0.67-2.37)$ & 0.48 \\
\hline Tumour loc & & & $0.05^{*}$ & & & \\
\hline Lateral & 1 & - & & $£$ & - & \\
\hline Medial & 1.10 & $(1.01-1.21)$ & 0.03 & - & - & \\
\hline Other & 1.07 & $(1.00-1.16)$ & 0.06 & - & - & \\
\hline Final op & & & - & & & \\
\hline $\mathrm{BCS}$ & 1.20 & $(1.07-1.34)$ & $<0.01$ & $£$ & - & \\
\hline Surg. marg. $¥$ & & & $<0.01^{*}$ & & & \\
\hline NM & 1 & - & & $£$ & - & \\
\hline FPM & 1.19 & $(1.02-1.38)$ & 0.03 & - & - & \\
\hline MFPM & 1.85 & $(1.43-2.40)$ & $<0.01$ & - & - & \\
\hline Adj. therapy & & & $<0.01 *$ & & & $<0.01 *$ \\
\hline Radio-yes & 0.45 & $(0.40-0.50)$ & $<0.01$ & 0.48 & $(0.34-0.69)$ & $<0.01$ \\
\hline Chemo-yes & 0.63 & $(0.57-0.70)$ & $<0.01$ & 0.65 & $(0.38-1.11)$ & 0.11 \\
\hline
\end{tabular}


Table 3 (continued)

\begin{tabular}{|c|c|c|c|c|c|c|}
\hline \multirow[t]{3}{*}{ Variable } & \multicolumn{3}{|c|}{ Overall survival } & \multicolumn{3}{|c|}{ Disease-free survival } \\
\hline & \multicolumn{3}{|c|}{ Total study population } & \multicolumn{3}{|c|}{ Total study population } \\
\hline & HR & $95 \%-\mathrm{CI}$ & $P$-value & HR & $95 \%-\mathrm{CI}$ & $P$-value \\
\hline Horm.-yes & 0.74 & $(0.67-0.81)$ & $<0.01$ & 0.56 & $(0.33-0.97)$ & 0.04 \\
\hline Target—yes & 0.50 & $(0.41-0.62)$ & $<0.01$ & 0.51 & $(0.23-1.10)$ & 0.09 \\
\hline \multirow[t]{3}{*}{ Variable } & \multicolumn{3}{|c|}{ Overall survival } & \multicolumn{3}{|c|}{ Disease-free survival } \\
\hline & \multicolumn{3}{|c|}{ Invasive carcinoma NST } & \multicolumn{3}{|c|}{ Invasive carcinoma NST } \\
\hline & HR & $95 \%-\mathrm{CI}$ & $P$-value & $\overline{\mathrm{HR}}$ & $95 \%$-CI & $P$-value \\
\hline \multicolumn{7}{|l|}{$\mathrm{b}$} \\
\hline$M R I$ & & & - & & & - \\
\hline Yes & 0.96 & $(0.78-1.19)$ & 0.74 & 1.23 & $(0.82-1.83)$ & 0.32 \\
\hline Age & & & $<0.01 *$ & & & $<0.01 *$ \\
\hline$<50$ & 1 & - & & 1 & - & \\
\hline $50-59$ & 1.28 & $(1.06-1.53)$ & $<0.01$ & 1.30 & $(0.74-2.30)$ & 0.36 \\
\hline $60-69$ & 1.79 & $(1.51-2.13)$ & $<0.01$ & 1.78 & $(1.03-3.07)$ & 0.04 \\
\hline$>70$ & 3.49 & $(2.94-4.15)$ & $<0.01$ & 2.67 & $(1.57-4.53)$ & $<0.01$ \\
\hline MRI\#age & & & $0.07 *$ & & & \\
\hline$<50$ & 1 & - & & - & - & \\
\hline $50-59$ & 0.85 & $(0.63-1.14)$ & 0.28 & - & - & \\
\hline $60-69$ & 0.83 & $(0.63-1.10)$ & 0.19 & - & - & \\
\hline$>70$ & 0.69 & $(0.53-0.91)$ & $<0.01$ & - & - & \\
\hline Tumour status & & & $<0.01 *$ & & & $<0.01 *$ \\
\hline 1 & 1 & - & & 1 & - & \\
\hline 2 & 1.74 & $(1.60-1.89)$ & $<0.01$ & 2.52 & $(1.70-3.76)$ & $<0.01$ \\
\hline $3-4$ & 2.33 & $(1.97-2.76)$ & $<0.01$ & 4.08 & $(1.90-8.78)$ & $<0.01$ \\
\hline Nodal status & & & $<0.01 *$ & & & $<0.01 *$ \\
\hline 0 & 1 & - & & 1 & - & \\
\hline 1 & 1.66 & $(1.52-1.81)$ & $<0.01$ & 1.57 & (1.03-2.39) & 0.03 \\
\hline 2 & 3.55 & $(3.08-4.10)$ & $<0.01$ & 4.09 & $(2.24-7.48)$ & $<0.01$ \\
\hline 3 & 5.98 & (5.09-7.03) & $<0.01$ & 8.86 & $(4.88-16.09)$ & $<0.01$ \\
\hline Hist. grade & & & $<0.01 *$ & & & $<0.01 *$ \\
\hline Low & 1 & - & & 1 & - & \\
\hline Medium & 1.26 & $(1.13-1.41)$ & $<0.01$ & 1.71 & $(0.86-3.42)$ & 0.13 \\
\hline High & 1.97 & $(1.74-2.23)$ & $<0.01$ & 3.92 & $(1.97-7.80)$ & $<0.01$ \\
\hline \multicolumn{7}{|l|}{ Multifocal } \\
\hline Yes & $£$ & - & & $£$ & - & \\
\hline Mol. subtype & & & $<0.01^{*}$ & & & $0.40^{*}$ \\
\hline ER/PR + HER2- & 1 & - & & 1 & - & \\
\hline ER/PR + HER2 + & 1.26 & $(1.08-1.47)$ & $<0.01$ & 1.73 & $(0.88-3.37)$ & 0.11 \\
\hline HER2 enr & 1.43 & $(1.18-1.73)$ & $<0.01$ & 1.68 & $(0.69-4.11)$ & 0.26 \\
\hline Triple neg & 1.71 & $(1.50-1.94)$ & $<0.01$ & 1.18 & $(0.61-2.27)$ & 0.62 \\
\hline Tumour loc & & & $0.01 *$ & & & \\
\hline Lateral & 1 & - & & $£$ & - & \\
\hline Medial & 1.12 & $(1.02-1.23)$ & 0.02 & - & - & \\
\hline Other & 1.11 & $(1.02-1.20)$ & 0.01 & - & - & \\
\hline Final op & & & - & & & \\
\hline BCS & 1.27 & $(1.12-1.43)$ & $<0.01$ & $£$ & - & \\
\hline
\end{tabular}


Table 3 (continued)

\begin{tabular}{|c|c|c|c|c|c|c|}
\hline \multirow[t]{3}{*}{ Variable } & \multicolumn{3}{|c|}{ Overall survival } & \multicolumn{3}{|c|}{ Disease-free survival } \\
\hline & \multicolumn{3}{|c|}{ Invasive carcinoma NST } & \multicolumn{3}{|c|}{ Invasive carcinoma NST } \\
\hline & HR & $95 \%-\mathrm{CI}$ & $P$-value & HR & $95 \%-\mathrm{CI}$ & $P$-value \\
\hline Surg. marg. $¥$ & & & $<0.01^{*}$ & & & \\
\hline NM & 1 & - & & $£$ & - & \\
\hline FPM & 1.23 & $(1.05-1.45)$ & 0.01 & - & - & \\
\hline MFPM & 1.88 & $(1.38-2.56)$ & $<0.01$ & - & - & \\
\hline Adj. therapy & & & $<0.01 *$ & & & $<0.01^{*}$ \\
\hline Radio-yes & 0.43 & $(0.38-0.48)$ & $<0.01$ & 0.43 & $(0.30-0.63)$ & $<0.01$ \\
\hline Chemo-yes & 0.65 & $(0.58-0.73)$ & $<0.01$ & $£$ & - & \\
\hline Horm.- -yes & 0.74 & $(0.67-0.82)$ & $<0.01$ & 0.57 & $(0.32-1.02)$ & 0.06 \\
\hline Target-yes & 0.45 & $(0.37-0.56)$ & $<0.01$ & 0.44 & $(0.21-0.95)$ & 0.04 \\
\hline \multirow[t]{3}{*}{ Variable } & \multicolumn{3}{|c|}{ Overall survival } & \multicolumn{3}{|c|}{ Disease-free survival } \\
\hline & \multicolumn{3}{|l|}{ ILC } & \multicolumn{3}{|l|}{ ILC } \\
\hline & HR & $95 \%$-CI & $P$-value & HR & $95 \%$-CI & $P$-value \\
\hline \multicolumn{7}{|l|}{$\mathrm{c}$} \\
\hline$M R I$ & & & - & & & - \\
\hline Yes & 0.54 & $(0.23-1.24)$ & 0.15 & 1.02 & $(0.36-2.94)$ & 0.96 \\
\hline Age & & & $<0.01 *$ & & & $0.48 *$ \\
\hline$<50$ & 1 & - & & 1 & - & \\
\hline $50-59$ & 0.92 & $(0.40-2.10)$ & 0.85 & 1.97 & $(0.36-10.82)$ & 0.43 \\
\hline $60-69$ & 1.45 & $(0.71-2.98)$ & 0.31 & 0.88 & $(0.14-5.40)$ & 0.89 \\
\hline$>70$ & 3.72 & $(1.83-7.56)$ & $<0.01$ & 0.59 & $(0.09-3.73)$ & 0.57 \\
\hline MRI\#age & & & $0.23^{*}$ & & & \\
\hline$<50$ & 1 & - & & - & - & \\
\hline $50-59$ & 1.67 & $(0.59-4.74)$ & 0.33 & - & - & \\
\hline $60-69$ & 1.67 & $(0.66-4.19)$ & 0.28 & - & - & \\
\hline$>70$ & 1.10 & $(0.46-2.64)$ & 0.84 & - & - & \\
\hline Tumour status & & & $<0.01 *$ & & & $0.31 *$ \\
\hline 1 & 1 & - & & 1 & - & \\
\hline 2 & 1.39 & $(1.10-1.75)$ & $<0.01$ & 2.83 & $(0.73-11.03)$ & 0.13 \\
\hline $3-4$ & 2.02 & $(1.50-2.72)$ & $<0.01$ & 2.82 & $(0.53-15.14)$ & 0.23 \\
\hline Nodal status & & & $<0.01 *$ & & & $<0.01 *$ \\
\hline 0 & 1 & - & & 1 & - & \\
\hline 1 & 1.67 & $(1.33-2.11)$ & $<0.01$ & 2.77 & $(0.72-10.66)$ & 0.14 \\
\hline 2 & 3.02 & $(2.09-4.36)$ & $<0.01$ & 19.39 & (3.16-118.88) & $<0.01$ \\
\hline 3 & 6.11 & $(4.59-8.13)$ & $<0.01$ & 28.69 & $(5.75-143.08)$ & $<0.01$ \\
\hline Hist. grade & & & $<0.01 *$ & & & $0.17 *$ \\
\hline Low & 1 & - & & 1 & - & \\
\hline Medium & 1.28 & $(0.95-1.71)$ & & 2.57 & $(0.40-16.57)$ & 0.32 \\
\hline High & 1.92 & $(1.32-2.78)$ & & 6.86 & $(0.75-62.65)$ & 0.09 \\
\hline \multicolumn{7}{|l|}{ Multifocal } \\
\hline Yes & $£$ & - & & $£$ & - & \\
\hline Mol. subtype & & & $0.04 *$ & & & - \\
\hline ER/PR + HER2- & 1 & - & & 1 & - & \\
\hline $\mathrm{ER} / \mathrm{PR}+\mathrm{HER} 2+$ & 1.10 & $(0.66-1.84)$ & 0.72 & - & - & \\
\hline HER2 enr & 1.59 & $(0.67-3.78)$ & 0.30 & - & - & \\
\hline Triple neg & 1.85 & $(1.20-2.86)$ & $<0.01$ & 1.08 & $(0.08-14.30)$ & 0.95 \\
\hline Tumour loc & & & & & & \\
\hline
\end{tabular}


Table 3 (continued)

\begin{tabular}{|c|c|c|c|c|c|c|}
\hline \multirow[t]{3}{*}{ Variable } & \multicolumn{3}{|c|}{ Overall survival } & \multicolumn{3}{|c|}{ Disease-free survival } \\
\hline & \multicolumn{3}{|l|}{ ILC } & \multicolumn{3}{|l|}{ ILC } \\
\hline & HR & $95 \%$-CI & $P$-value & HR & $95 \%-\mathrm{CI}$ & $P$-value \\
\hline Lateral & $£$ & - & & $£$ & - & \\
\hline Medial & - & - & & - & - & \\
\hline Other & - & - & & - & - & \\
\hline \multicolumn{7}{|l|}{ Final op } \\
\hline BCS & $£$ & - & & $£$ & - & \\
\hline \multicolumn{7}{|l|}{ Surg. marg. $¥$} \\
\hline NM & $£$ & - & & 1 & - & \\
\hline FPM & - & - & & 1.82 & $(0.34-9.75)$ & 0.48 \\
\hline MFPM & - & - & & 11.91 & $(0.61-234.14)$ & 0.10 \\
\hline Adj. therapy & & & $<0.01 *$ & & & $0.03^{*}$ \\
\hline Radio-yes & 0.54 & $(0.45-0.66)$ & $<0.01$ & $£$ & - & \\
\hline Chemo-yes & 0.56 & $(0.41-0.76)$ & $<0.01$ & 0.20 & $(0.04-0.93)$ & 0.04 \\
\hline Horm.-yes & 0.73 & $(0.57-0.94)$ & 0.01 & 0.31 & $(0.07-1.30)$ & 0.11 \\
\hline Target-yes & $£$ & - & & $£$ & - & \\
\hline
\end{tabular}

NST no special type, ILC invasive lobular carcinoma, MRI magnetic resonance imaging, $H R$ hazard ratio, ¥ final operation, MRI\#age interaction term MRI and age per category, only computed for OS-cohort, Hist. grade histological grade, Mol. Subtype molecular subtype, HER2 enr. HER2 enriched, Triple neg. triple negative, Tumour loc. tumour location, Final op. final operation, BCS breast conserving surgery, Surg. Marg. surgical margin, NM negative margin, FPM focal positive margin, MFPM more than focal positive margin, Adj. therapy adjuvant therapy, Horm. Hormonal

$£$ excluded due to Akaike's information criterion selection method; ${ }^{*} P$-value overall likelihood ratio test

subgroup of patients with regard to potential change in treatment decision based on breast MRI findings [19].

In addition, the patient characteristics and treatment characteristics within our observational study were not well balanced. After further examination of the treatment characteristics of the OS study population, it was somewhat noticeable that patients in the total study population and in the age categories $>60$ treated with mastectomy, were slightly more treated with adjuvant radiotherapy in the MRI group, compared to the non-MRI group. Patients in the age category of $>70$ and treated with breast conserving surgery, were slightly less treated with adjuvant radiotherapy in the non-MRI group, compared to the MRI group. In both the invasive carcinoma NST subgroup and the ILC subgroup the same results applied, however in the invasive carcinoma NST subgroup this was less noticeable. In addition, in the ILC subgroup patients in the age category of $<50$ and treated with breast conserving surgery, were slightly less treated with adjuvant radiotherapy in the non-MRI group, compared to the MRI group. Although we corrected for the unbalanced patient and treatment characteristics in our multivariable analysis, we still cannot exclude residual confounding.

Another limitation is the maximum follow-up of 7 years, which resulted in a mean follow-up of 5.3 years. A longer follow-up would have given more insight into the possible long-term impact of breast MRI on the OS, especially with regard to hormone receptor positive patients, and would have increased the statistical power to detect clinically relevant differences. In addition, the cause of death was unknown in the current study, consequently breast cancer related mortality cannot be evaluated in the current cohort. In order to provide a better recommendation for breast MRI in general use, it is recommended that a next study should focus on a longer follow-up period, including a breast cancer related mortality analysis.

Finally, our DFS-cohort consisted only of a relatively small proportion of the patients out of the OS-cohort. Due to limited resources active follow-up performed by the registrars of the NCR could only be accomplished for the patients diagnosed in the first quartile of 2012.

Future studies should investigate the effect on prognosis by comparing patients in whom MRI findings led to a change in treatment plan with those without treatment change and/or patients without MRI. For instance, the currently recruiting European wide MIPA trial could identify this specific subgroup of patients allowing to investigate prognosis in these patients into detail [20].

In summary, use of breast MRI showed no statistically significant effect regarding DFS nor OS for different age categories in patients treated with primary surgery. 
Funding This study did not receive any funding.

\section{Compliance with ethical standards}

Conflict of interest The authors declare that they have no conflicts of interest.

Ethical approval All procedures performed in studies involving human participants were in accordance with the ethical standards of the institutional and/or national research committee and with the 1964 Helsinki declaration and its later amendments or comparable ethical standards.

Informed consent All patient data were received anonymously in this study (without patient information) from the Netherlands Cancer Registry (NCR). According to the Dutch law, all cancer patients are included in the NCR as maintained by the Netherlands Comprehensive Cancer Organisation (IKNL), unless the patient objected to be registered. Consequently, informed consent was not applicable for this study.

Open Access This article is licensed under a Creative Commons Attribution 4.0 International License, which permits use, sharing, adaptation, distribution and reproduction in any medium or format, as long as you give appropriate credit to the original author(s) and the source, provide a link to the Creative Commons licence, and indicate if changes were made. The images or other third party material in this article are included in the article's Creative Commons licence, unless indicated otherwise in a credit line to the material. If material is not included in the article's Creative Commons licence and your intended use is not permitted by statutory regulation or exceeds the permitted use, you will need to obtain permission directly from the copyright holder. To view a copy of this licence, visit http://creativecommons.org/licenses/by/4.0/.

\section{References}

1. World Health Organization (2018) Cancer: key facts. World Health Organization. https://www.who.int/news-room/fact-sheets/detail/ cancer. Accessed 15 Aug 2020

2. Integraal Kankercentrum Nederland (2019) Cijfers over kankerIncidentie. https://www.cijfersoverkanker.nl/selecties/incidentie _borst/img5ba0dbccdd287. Accessed 15 Aug 2020

3. Lobbes MBI et al (2017) Breast MRI increases the number of mastectomies for ductal cancers, but decreases them for lobular cancers. Breast Cancer Res Treat 162:353-364

4. Houssami N, Turner R, Morrow M (2013) Preoperative magnetic resonance imaging in breast cancer. Ann Surg 257:249-255

5. Gruber IV et al (2013) Measurement of tumour size with mammography, sonography and magnetic resonance imaging as compared to histological tumour size in primary breast cancer. BMC Cancer 13:1

6. Plana MN et al (2012) Magnetic resonance imaging in the preoperative assessment of patients with primary breast cancer: Systematic review of diagnostic accuracy and meta-analysis. Eur Radiol 22:26-38

7. Vapiwala N, Hwang W, Kushner CJ, Schnall MD (2017) No impact of breast magnetic resonance imaging on 15-year outcomes in patients with ductal carcinoma in situ or early-stage invasive breast cancer managed with breast conservation therapy. Cancer. https:// doi.org/10.1002/cncr.30479

8. Vos EL et al (2015) Benefits of preoperative MRI in breast cancer surgery studied in a large population-based cancer registry. Br J Surg 102:1649-1657

9. Houssami $\mathrm{N}$ et al (2014) An individual person data meta-analysis of preoperative magnetic resonance imaging and breast cancer recurrence. J Clin Oncol 32:392

10. Sung JS et al (2014) Preoperative breast MRI for early-stage breast cancer: effect on surgical and long-term outcomes. Am J Roentgenol. https://doi.org/10.2214/AJR.13.11355

11. Choi WJ et al (2017) Long-term survival outcomes of primary breast cancer in women with or without preoperative magnetic resonance imaging: a matched cohort study. Clin Oncol 29:653-661

12. Ryu J et al (2016) Preoperative magnetic resonance imaging and survival outcomes in T1-2 breast cancer patients who receive breastconserving therapy. J Breast Cancer 19:423

13. Solin LJ, Orel SG, Hwang WT, Harris EE, Schnall MD (2008) Relationship of breast magnetic resonance imaging to outcome after breast-conservation treatment with radiation for women with earlystage invasive breast carcinoma or ductal carcinoma in situ. J Clin Oncol 26:386-391

14. Ha SM, Chae EY (2019) Long-term survival outcomes in invasive lobular carcinoma patients with and without preoperative MR imaging: a matched cohort study. Eur Radiol 29:2526

15. Sobin LH, Gospodarowicz MK, Wittekind C (2010) TNM classification of malignant tumours. Blackwell Publishing Ltd, Hoboken

16. Integraal Kankercentrum Nederland (2012) Mammacarcinoom: Landelijke richtlijn. https://heelkunde.nl/sites/heelkunde.nl/files/ richtlijnen-definitief/Mammacarcinoom2012.pdf. Accessed 15 Aug 2020

17. White IR et al (2011) Multiple imputation using chained equations: issues and guidance for practice. Stat Med 30(4):377-399

18. Lai H et al (2018) Clinicopathologic factors related to surgical margin involvement, reoperation, and residual cancer in primary operable breast cancer - an analysis of 2050 patients. Eur J Surg Oncol 44:1725-1735

19. Mann RM et al (2015) Breast MRI: EUSOBI recommendations for women's information. Eur Radiol 25(12):3669-3678

20. Sardanelli F et al (2020) Solving the preoperative breast MRI conundrum: design and protocol of the MIPA study. Eur Radiol. https:// doi.org/10.1007/s00330-020-06824-7

Publisher's Note Springer Nature remains neutral with regard to jurisdictional claims in published maps and institutional affiliations. 


\section{Affiliations}

\section{T. J. A. van Nijnatten ${ }^{1,2}$ - L. P. T. van Tiel ${ }^{3} \cdot$ A. C. Voogd $d^{2,4,5}$. C. G. M. Groothuis-Oudshoorn ${ }^{3} \cdot$ S. Siesling ${ }^{3,4}$. M. B. I. Lobbes ${ }^{1,2,6}$}

1 Department of Radiology and Nuclear Medicine, Maastricht University Medical Center+, P.O. Box 5800, 6202 AZ Maastricht, The Netherlands

2 GROW - School for Oncology and Developmental Biology, Maastricht University Medical Center+, Maastricht, The Netherlands

3 Department of Health Technology and Services Research, Technical Medical Center, University of Twente, Enschede, The Netherlands
4 Department of Research and Development, Netherlands Comprehensive Cancer Organisation, Utrecht, The Netherlands

5 Department of Epidemiology, Maastricht University Medical Center+, Maastricht, The Netherlands

6 Department of Medical Imaging, Zuyderland Medical Center, Sittard-Geleen, The Netherlands 